\title{
Enhanced Expression of Hemoglobin Scavenger Receptor CD163 in Accumulated Macrophages Within Filtered Debris Between Acute Coronary Syndromes and Stable Angina Pectoris
}

\author{
Takao SATo, ${ }^{1} \mathrm{MD}$, Tomoki KAmeyama, ${ }^{1} \mathrm{MD}$, Takahisa Noto, ${ }^{2} \mathrm{MD}$, \\ Hiroshi Ueno, ${ }^{1} \mathrm{MD}$, and Hiroshi Inoue, ${ }^{1} \mathrm{MD}$
}

\begin{abstract}
SUMMARY
Coronary intraplaque hemorrhage up-regulates hemoglobin scavenger receptor CD163 expression on macrophages, and has an association with vulnerable plaque development. During percutaneous coronary intervention, mechanical plaque disruption exposes potentially embolic atheromatous contents from culprit plaque.

In 37 patients with stable angina pectoris (SAP, $n=20$ ) or acute coronary syndrome (ACS, $n=17$ ), atherothrombotic debris was collected using a filter-based distal embolic protection device. We immunohistochemically determined CD14-positive macrophages and CD163-positive macrophages in filtered debris. We also examined the relation of CD14- and CD163-positive macrophages with culprit plaque volume and components evaluated with ultrasonic tissue characterization (VH-IVUS).

The only significant difference in clinical characteristics between the two groups was in hs-CRP. In ACS, the percentage of CD14- and CD163-positive macrophages to the whole cells (\%CD14 and \%CD163, respectively) was significantly higher than that in SAP $(20.1 \pm 8.2$ versus $8.8 \pm 6.8 \%, P<0.001$ and $32.6 \pm 18.9$ versus $9.0 \pm 3.8 \%, P<0.001$, respectively). In IVUS indices of culprit plaque, the remodeling index was significantly higher in ACS than in SAP. However, necrotic core component $(\% \mathrm{NC})$ in ACS was significantly higher than that in SAP. Furthermore, fibrotic component (\%Fibrous) in ACS was significantly lower than that in SAP $(56.1 \pm 4.7$ versus $60.1 \pm 3.3 \%, P=0.03) . \%$ CD14 and \%CD163 had a significant positive correlation with \%NC (\%CD14: $r=0.40, P=0.01$ and \%CD163: $r=0.45, P=$ $0.01)$, but only \%CD163 was negatively correlated with \%Fibrous (\%CD163: $r=-0.48, P=0.01)$.

These findings suggest that the presence of CD14- and CD163-positive macrophages may reflect plaque inflammation, NC expansion, and plaque vulnerability in patients with coronary heart disease. (Int Heart J 2015; 56: 150-156)
\end{abstract}

Key words: Coronary artery disease, Intraplaque hemorrhage, Catheters

$\mathrm{B}$ oth macrophage infiltration and hemorrhage into coronary plaque are associated with the development of atherosclerotic lesions and plaque instability. ${ }^{1)}$ Atherosclerosis largely involves macrophage-mediated inflammation driven by oxidized lipids. ${ }^{2}$

CD14 on the macrophage surface is bound to lipopolysaccharides, thereby activating macrophages to produce cytokines such as TNF- $\alpha$, IL-1, and IL-6, which, in turn, serve as endogenous mediators of inflammation. ${ }^{3)}$ In a previous pathological report, CD14-positive macrophages were more prevalent in atherosclerotic lesions compared with normal intimal regions of coronary arteries. ${ }^{3)}$ Thus, CD 14-positive macrophages within the plaque indicates plaque with inflammatory macrophage inflammation.

In advanced atherosclerosis, intraplaque hemorrhage (IPH) is an important contributor to lesion development and destabilization by several mechanisms, including the release of hemoglobin $(\mathrm{Hb})$ into plaque. ${ }^{4)} \mathrm{Hb}$ derived from IPH binds tightly to haptoglobin $(\mathrm{Hp})$ to form an $\mathrm{Hp}-\mathrm{Hb}$ complex. The
$\mathrm{Hp}-\mathrm{Hb}$ complex is exclusively cleared by macrophages with a scavenger receptor, CD163, and IPH also induces accumulation of CD163-positive macrophages. ${ }^{5)} \mathrm{A}$ recent study indicated that IPH was seen more frequently in the coronary vasculature in patients dying from plaque rupture, a primary cause of acute coronary syndrome (ACS), as compared with plaque erosion or stable lesions. ${ }^{\text {.) }}$

Intravascular ultrasound (IVUS)-derived virtual histology (VH-IVUS), using spectral analysis of the radiofrequency ultrasound backscatter signals, could identify components of atherosclerotic plaque. ${ }^{7}$

During percutaneous coronary intervention (PCI), mechanical plaque disruption exposes potentially embolic atheromatous contents from the culprit plaque. Filter-based distal embolic protection devices can retrieve this atherothrombotic debris. ${ }^{8}$

The aim of the present study was to investigate the relation among CD14- and CD163-positive macrophage infiltration, culprit plaque morphology, and plaque vulnerability in

From the ${ }^{1}$ Second Department of Internal Medicine, University of Toyama, Toyama and ${ }^{2}$ Imizu Municipal Hospital, Imizu, Japan

Address for correspondence: Takao Sato, MD, The Second Department of Internal Medicine, Toyama University Hospital, 2630 Sugitani, Toyama, Toyama 930-0194, Japan. E-mail: kirotaro19731013@yahoo.co.jp

Received for publication July 8, 2014. Revised and accepted August 13, 2014.

Released in advance online on J-STAGE February 23, 2015.

All rights reserved by the International Heart Journal Association. 
patients with ACS and stable angina pectoris (SAP).

\section{Methods}

Patients: Fifty-five consecutive patients who underwent percutaneous coronary intervention (PCI) for ACS $(n=17)$ and SAP $(n=38)$ were enrolled. Patients had ischemic chest discomfort with ST-segment elevation or depression of $>0.5 \mathrm{~mm}$ or T-wave inversion in two or more leads. ACS was diagnosed by increased serum levels of creatine kinase (more than twice the upper limit of the normal) and creatine kinase-MB fraction ( $>10 \%$ of total creatine kinase). Patients without elevation of the creatine kinase-MB fraction were classified as having unstable angina pectoris (UPA). SAP was diagnosed by stress electrocardiology, radioisotope, or coronary angiography.

Patients were excluded if they had significant left main coronary artery disease, extremely tortuous or heavily calcified vessels, renal insufficiency with baseline serum creatinine $\geq 1.8$ $\mathrm{mg} / \mathrm{dL}$, or infectious or inflammatory diseases. The study protocol was approved by the institutional ethics committee of Toyama University Hospital and the participating institutions, and written informed consent was obtained from all patients.

Laboratory assessments: A blood sample was taken from the antecubital vein after a minimum fast of 12 hours, and serum blood glucose and lipid profiles [total cholesterol, triglycerides (TG), high-density lipoprotein cholesterol (HDL-C), low-density lipoprotein cholesterol (LDL-C)], HbA1c [National Glycohemoglobin Standardization Program (NGSP)] and high sensitivity-C reactive protein (hs-CRP) were determined.

Procedures and protocol: In patients with ACS, aspirin 100 mg was given orally in the emergency room. Additionally, all patients were given clopidogrel at a loading dose of $300 \mathrm{mg}$ before PCI, and in patients with SAP, aspirin $100 \mathrm{mg}$ and clopidogrel $75 \mathrm{mg}$ were administered orally from 1 week before PCI. Intravenous unfractionated heparin was also given to achieve an activated clotting time of 200-250 seconds.

Angiography was performed with a 6-F guiding catheter to identify the site of the culprit lesion. A 0.014 -inch coronary guidewire was passed through the lesion. Before PCI, aspiration of thrombi was performed with an aspiration thrombectomy catheter (Thrombuster III ${ }^{\circledR}$, KANEKA, Osaka, Japan), followed by placement of a filter-based distal protection device (Filtrap ${ }^{\circledR}$, NIPRO, Osaka, Japan), distal to the culprit lesion. All IVUS procedures were then performed after administration of intracoronary nitrates with a $20-\mathrm{MHz}, 2.9-\mathrm{F}$, phased-array IVUS catheter (Eagle Eye, Volcano Corporation, Rancho Cordova, CA, USA). All patients were treated by deployment of a bare-metal stent or drug-eluting stent to the culprit lesion. After completion of the PCI procedure, the Filtrap ${ }^{\circledR}$ was removed, and atherothrombotic debris was collected for immunohistological analyses.

IVUS imaging: All IVUS examinations were performed using a motorized pull-back system (R-100, Volcano Corporation) at a speed of $0.5 \mathrm{~mm} / \mathrm{second}$. Culprit lesions were defined according to electrocardiographic criteria (ST-segment shift or Twave inversion), wall-motion abnormalities by echocardiography and angiographic appearances (point of angiographic maximal stenosis, luminal irregularities consistent with ulceration, or filling defects consistent with thrombus) at PCI. The target artery was observed using IVUS before stent deploy- ment. During pullback, gray-scale IVUS was recorded, and raw radiofrequency data were captured at the top of the $\mathrm{R}$ wave of electrocardiographic monitoring for later reconstruction of a color-coded map by a VH-IVUS console (S5, Volcano Corporation).

Gray scale and VH-IVUS analyses: Quantitative analysis was performed according to the American College of Cardiology clinical expert consensus document on IVUS. ${ }^{9)}$ The lesion where the stent had been placed, identified using side branches and unique calcification as landmarks on IVUS imaging prior to stent implantation, was analyzed using IVUS. Cross-sectional area (CSA) of the lumen and vessel was determined at the reference as well as the culprit lesion site using automatic edge detection and corrected manually when necessary. Vessel CSA was determined with the external elastic membrane (EEM) as the outer margin of the vessel, and lumen CSA, with the lumen border as the outer margin of the lumen. The difference between vessel CSA and lumen CSA thus provided the CSA of the plaque + media. The percentage of the CSA of plaque + media was calculated as an index of plaque burden by dividing the CSA of the plaque + media by the vessel CSA. The proximal and distal reference segments were defined as the segments with the largest lumen with the least plaque and located within $5 \mathrm{~mm}$ proximal and distal to the lesion, but proximal to a major side branch. ${ }^{7)}$ Remodeling index (RI) was calculated by dividing EEM CSA at the culprit lesion by the mean of the proximal and distal reference CSA. Remodeling with an RI $>1.05$ was defined as positive remodeling, whereas remodeling with an $\mathrm{RI}<0.95$ was defined as negative remodeling. ${ }^{10)}$ Based on mathematical autoregressive spectral analysis of IVUS backscattered data, atherosclerotic coronary plaques were characterized as fibrous plaque (Fibrous), fibro-fatty plaque (FF), necrotic core (NC), and dense calcium (DC) as described previously. ${ }^{11)}$ On the reconstructed color-coded tissue map, fibrous areas were marked in green, FF in greenishyellow, DC in white, and NC in red. The relative amounts of the 4 plaque components in the tissue map were calculated. Gray scale and VH-IVUS analyses were performed by two experienced cardiologists who were blinded to the quantitative analysis data as well as the baseline clinical and lesion characteristics.

Atherothrombotic debris harvesting: After completion of the PCI procedure, the Filtrap ${ }^{\circledR}$ was removed from the patient and atherothrombotic debris was collected for immunohistological analysis.

Quantification of immunohistological staining: The retrieved atherothrombotic debris was fixed in $10 \%$ formalin for 24 hours and embedded in paraffin. Four-micron-thick sections of the debris were cut at the largest volume site and stained with hematoxylin-eosin. To determine macrophage infiltration, mouse anti-human CD14 and CD163 monoclonal antibody (Novocastra Corporation, Newcastle Upon Tyne, UK) was used.

Immunohistochemical detection of the preferred epitopes was performed according to the indirect horseradish peroxidase technique. The tissue was incubated with primary antibodies followed by secondary antibodies (Envision, Dako Corporation) for anti-CD14 and CD163. Slides were developed with diaminobenzidine (Dako Corporation) and counterstained with REAL hematoxylin (Dako Corporation). All slides were digitized for histomorphometric analysis. The percentage of 
CD14- and CD163-positive macrophages to all cells per square millimeter (\%CD14 and \%CD163, respectively) was quantified (at $\times 20$ magnification) with an automatic membrane v9 Scan Scope CS system (Aperio, Leica Biosystems, Vista, CA, USA). ${ }^{8)}$

Statistical analysis: All statistical analyses were performed using the Sigma-Plot software program (version 11, Systat Software, Inc., San Jose, CA, USA). The data are presented as the mean \pm SD. Comparison between the two groups (ACS versus $\mathrm{SAP})$ was performed by using the unpaired $t$-test for continuous data and $\chi^{2}$ or Fisher's exact test for categorical data. Correlations of two variables were examined using Spearman's correlation analysis. A $P$ value of $<0.05$ was considered significant.

\section{RESULTS}

In the ACS group, atherothrombotic debris was successfully retrieved from all patients, while in the SAP group, 20 patients in whom atherothrombotic debris was successfully retrieved constituted the study group (Figure 1). In Figure 2, representative immunohistological staining of plaque debris is shown. The clinical, IVUS, and VH-IVUS characteristics of the patients are shown in Tables II, III, and IV. There were no significant differences in age, sex, body weight, hypertension, lipid profile, or glycemic control between the two treatment groups, but there was a significant difference in the hs-CRP level $(1.9 \pm 1.7$ versus $0.48 \pm 0.68 \mathrm{mg} / \mathrm{dL}, P=0.01)$ (Table I). In the comparison of IVUS parameters of culprit plaque between the ACS and SAP groups, the remodeling index was significantly higher in the ACS group than in the SAP group $(1.13 \pm 0.06$ versus $1.02 \pm 0.09, P=0.02)$ (Table II). However, a comparison of VH-IVUS parameters in culprit plaques between the ACS and SAP groups revealed a significant increase in \% NC and in NC volume in the ACS group, as well as a decrease in \%Fibrous, compared to the SAP group (Table III). The \%CD14-positive macrophages and \%CD163-positive macrophages in the ACS group were significantly higher than in the SAP group $(20.1 \pm 8.2$ versus $8.8 \pm 6.8 \%, P<0.001$ and $32.6 \pm 18.9$ versus $9.0 \pm 3.8 \%, P<0.001$, respectively) (Table IV). Furthermore, \%CD14- and \%CD163-positive macrophages had significant positive correlations with \%NC (\%CD14: $r$ $=0.40, P=0.01$ and \%CD163: $r=0.45, P=0.01)$ while only $\%$ CD163 was negatively correlation with \%Fibrous $(r=-0.48$, $P=0.01$ ) (Figure 3 ). Additionally, the hs-CRP level was significantly correlated with \%CD163-positive macrophages (CD163: $r=0.31, P=0.04$ ) (Figure 4).

\section{Discussion}

The major findings of the present study were as follows. First, the \%CD14 and \%CD163 levels in the ACS group were significantly higher than those in the SAP group; \%CD163 had a positive correlation with hs-CRP level. Second, the ACS group not only had significantly increased \% NC and NC volume, but also showed significantly decreased \%Fibrous compared to the SAP group. Finally, \%CD14 and \%CD163 were positively correlated with $\% \mathrm{NC}$, but only $\% \mathrm{CD} 163 \mathrm{had}$ a negative correlation with \%Fibrous in culprit plaques.

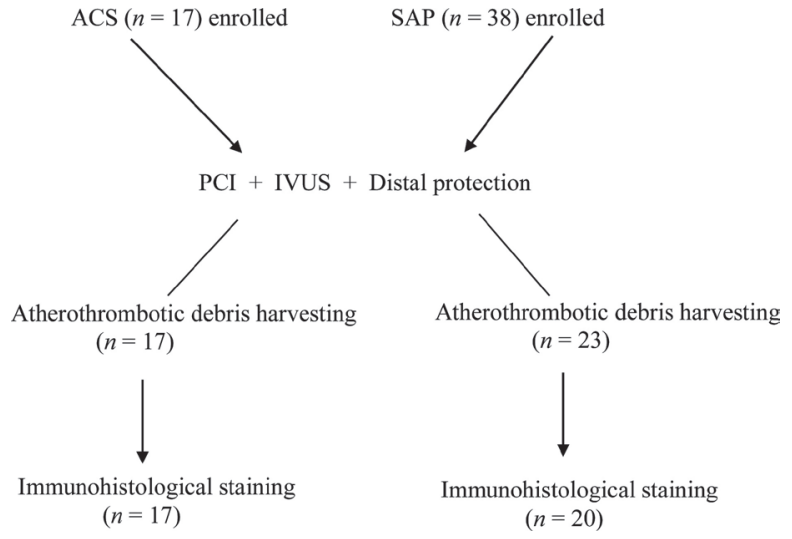

Figure 1. Group allocation.
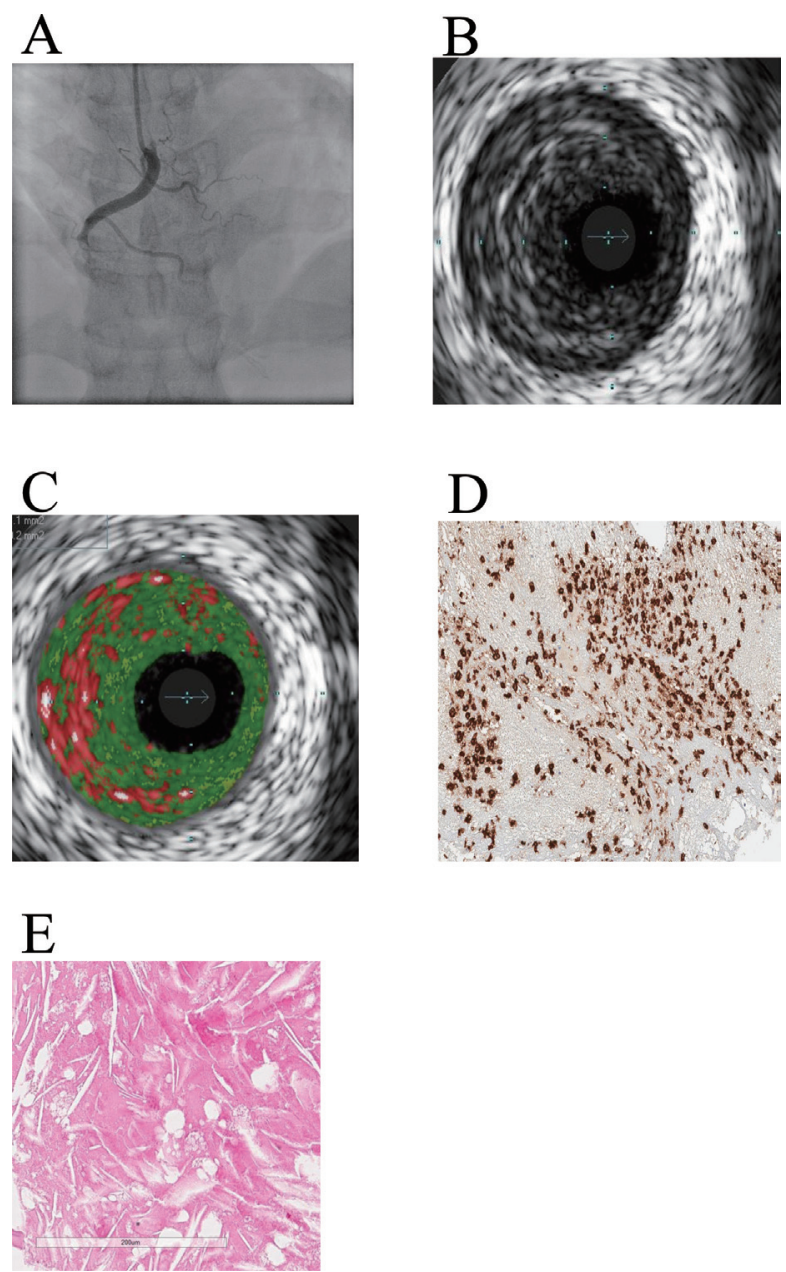

Figure 2. Immunohistological staining. A 49-year-old male patient with acute coronary syndrome who had $100 \%$ occlusion in the right coronary artery is shown (A). IVUS (B) was performed, and VH-IVUS (C) revealed a culprit plaque with a large necrotic core $(\% \mathrm{NC}, 30.3 \%)$ without calcification (\%DC, 3.4\%). Atherothrombotic plaque debris retrieved by a Fil$\operatorname{trap}^{\circledR}$ was immunostained for the macrophage marker CD163 (D). Plaque debris was abundant in CD163-positive macrophages (brown) (\%CD163, 88.6\%) and cholesterol crystals (E). 
Table I. Baseline Clinical Characteristics

\begin{tabular}{|c|c|c|c|c|}
\hline & All & $\mathrm{ACS}$ & SAP & $P(\mathrm{ACS}$ versus $\mathrm{SAP})$ \\
\hline Number & 37 & 17 & 20 & \\
\hline Age (years) & $70.1 \pm 14.1$ & $65.1 \pm 16.8$ & $73.7 \pm 10.8$ & NS \\
\hline Male & 26 & $10(59)$ & $16(80)$ & NS \\
\hline BMI $\left(\mathrm{kg} / \mathrm{m}^{2}\right)$ & $23.3 \pm 3.0$ & $22.7 \pm 3.0$ & $23.7 \pm 3.1$ & NS \\
\hline sBP (mmHg) & $132 \pm 26$ & $137 \pm 25$ & $129 \pm 26$ & NS \\
\hline $\mathrm{dBP}(\mathrm{mmHg})$ & $78 \pm 14$ & $80 \pm 16$ & $76 \pm 13$ & NS \\
\hline \multicolumn{5}{|l|}{ Glycemic control } \\
\hline FBS (mg/dL) & $126 \pm 43$ & $137 \pm 46$ & $119 \pm 35$ & NS \\
\hline HbA1c $(\%)$ & $6.2 \pm 1.5$ & $6.4 \pm 1.9$ & $6.0 \pm 1.3$ & NS \\
\hline \multicolumn{5}{|l|}{ Lipid profile } \\
\hline LDL (mg/dL) & $98 \pm 32$ & $99 \pm 40$ & $98 \pm 23$ & NS \\
\hline TG (mg/dL) & $120 \pm 52$ & $105 \pm 50$ & $131 \pm 53$ & NS \\
\hline $\mathrm{HDL}(\mathrm{mg} / \mathrm{dL})$ & $44 \pm 13$ & $47 \pm 15$ & $41 \pm 10.4$ & NS \\
\hline $\mathrm{CRP}(\mathrm{mg} / \mathrm{dL})$ & $1.2 \pm 1.4$ & $1.9 \pm 1.7$ & $0.5 \pm 0.7$ & 0.01 \\
\hline
\end{tabular}

Figures are number $(\%)$ or mean \pm SD. BMI indicates body mass index; sBP, systolic blood pressure; dBP, diastolic blood pressure; FBS, fasting blood sugar; LDL, low density lipoprotein cholesterol; TG, triglycerides; HDL, high density lipoprotein cholesterol; CRP, C-reactive protein; and NS, not significant.

Table II. Comparison of IVUS Parameters Between ACS and SAP

\begin{tabular}{lcccc}
\hline & All & ACS & SAP & $P($ ACS versus SAP) \\
\hline Vessel $\left(\mathrm{mm}^{2}\right)$ & $18.5 \pm 5.5$ & $19.0 \pm 4.4$ & $18.0 \pm 7.2$ & NS \\
Lumen $\left(\mathrm{mm}^{2}\right)$ & $4.4 \pm 1.3$ & $4.5 \pm 0.6$ & $4.2 \pm 1.6$ & NS \\
Plaque $\left(\mathrm{mm}^{2}\right)$ & $10.9 \pm 3.7$ & $10.9 \pm 4.8$ & $10.5 \pm 2.5$ & NS \\
\%Plaque & $75.6 \pm 3.5$ & $74.6 \pm 3.6$ & $76.7 \pm 3.0$ & NS \\
Remodeling index & $1.09 \pm 0.09$ & $1.13 \pm 0.06$ & $1.02 \pm 0.09$ & 0.02 \\
Stent diameter $(\mathrm{mm})$ & $3.2 \pm 0.3$ & $3.3 \pm 0.2$ & $3.1 \pm 0.3$ & NS \\
Stent length $(\mathrm{mm})$ & $16.4 \pm 4.4$ & $15.0 \pm 2.5$ & $17.6 \pm 4.1$ & NS \\
LAD/LCX/RCA $(n)$ & $18 / 11 / 8$ & $8 / 5 / 4$ & $10 / 6 / 4$ & NS \\
\hline
\end{tabular}

Figures are number or mean $\pm \mathrm{SD}$. LAD indicates left anterior descending; LCX, left circumflex; RCA, right coronary artery; and NS, not significant.

Table III. Comparison of VH-IVUS Data Between ACS and SAP

\begin{tabular}{lrcrc}
\hline & All & ACS & SAP & $P$ \\
\hline Fibrous $\left(\mathrm{mm}^{2}\right)(\%)$ & $6.5 \pm 2.6$ & $6.5 \pm 2.8$ & $6.4 \pm 2.3$ & NS \\
& $58.2 \pm 4.4$ & $56.1 \pm 4.7$ & $60.1 \pm 3.3$ & 0.03 \\
Fibro-fatty $\left(\mathrm{mm}^{2}\right)(\%)$ & $1.7 \pm 1.0$ & $1.7 \pm 1.1$ & $1.7 \pm 0.9$ & NS \\
& $15.0 \pm 6.7$ & $14.6 \pm 7.0$ & $15.1 \pm 7.2$ & NS \\
Necrotic core $\left(\mathrm{mm}^{2}\right)(\%)$ & $2.4 \pm 1.0$ & $2.7 \pm 1.2$ & $1.9 \pm 0.6$ & 0.04 \\
& $21.3 \pm 9.0$ & $24.6 \pm 10.2$ & $18.4 \pm 7.0$ & 0.04 \\
Dense calcium $\left(\mathrm{mm}^{2}\right)(\%)$ & $0.6 \pm 0.4$ & $0.5 \pm 0.3$ & $0.7 \pm 0.5$ & NS \\
& $5.5 \pm 4.3$ & $4.7 \pm 3.1$ & $6.4 \pm 5.4$ & $\mathrm{NS}$ \\
\hline
\end{tabular}

Figures are mean \pm SD. NS indicates not significant.

Table IV. Comparison of \%CD14 and \%CD163 Macrophage Between SAP and ACS

\begin{tabular}{lllll}
\hline & All & ACS & SAP & $P$ \\
\hline$\%$ CD14 & $14.0 \pm 9.3$ & $20.1 \pm 8.2$ & $8.8 \pm 6.8$ & $<0.001$ \\
$\%$ CD163 & $19.8 \pm 17.6$ & $32.6 \pm 18.9$ & $9.0 \pm 3.8$ & $<0.001$ \\
\hline
\end{tabular}

Figures are mean \pm SD.

IPH accelerates coronary atherosclerosis and evokes CD163positive macrophages: Atherosclerotic IPH is an important contributor to lesion development and destabilization. The mechanisms for atherogenicity of IPH include delivery of free cholesterol from the erythrocyte membrane, and the extravasation of inflammatory factors including macrophages and $\mathrm{Hb}$ - derived iron. Hb-derived iron can act as a catalyst in the formation of oxygen free-radicals. ${ }^{12,13)}$ In an autopsy study, IPH was seen most frequently in vulnerable thin-cap fibroatheroma (TCFA), leading to ACS, as compared with stable lesions. ${ }^{1)}$ This finding suggests IPH contributes to rapidly progressive plaque destabilization. The cell-free $\mathrm{Hb}$ produced by IPH is 

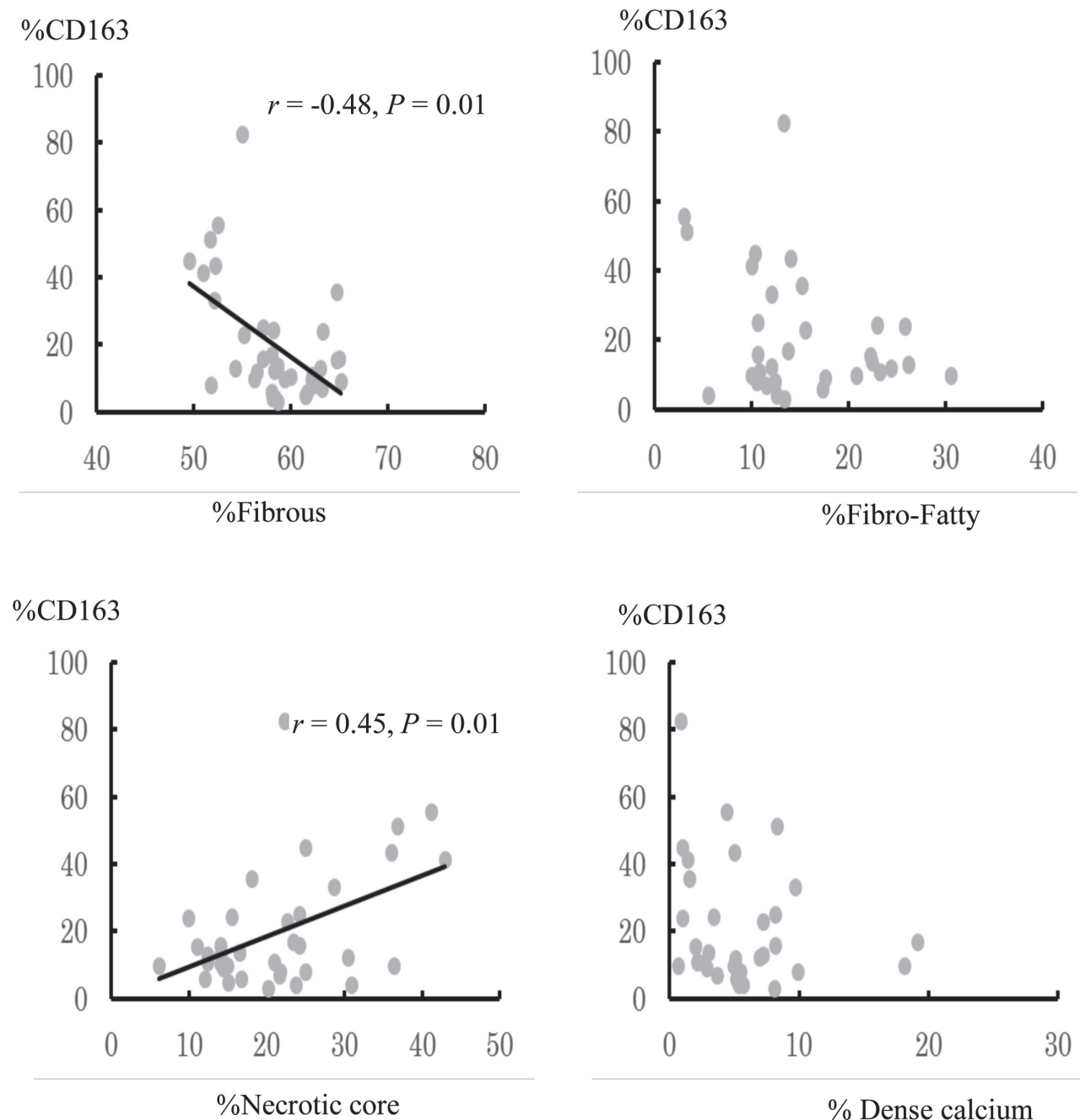

Figure 3. Correlation between percent of CD163 positive macrophages and VH-IVUS parameters (\%Fibrous, \%Fibro-fatty, \%Necrotic core and \%Dense calcium components). See text for details.

\section{$\% \mathrm{CD} 14$}

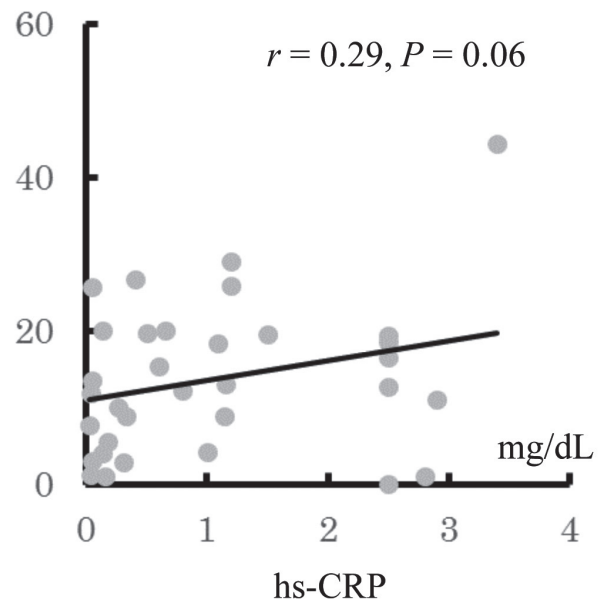

$\% \mathrm{CD} 163$

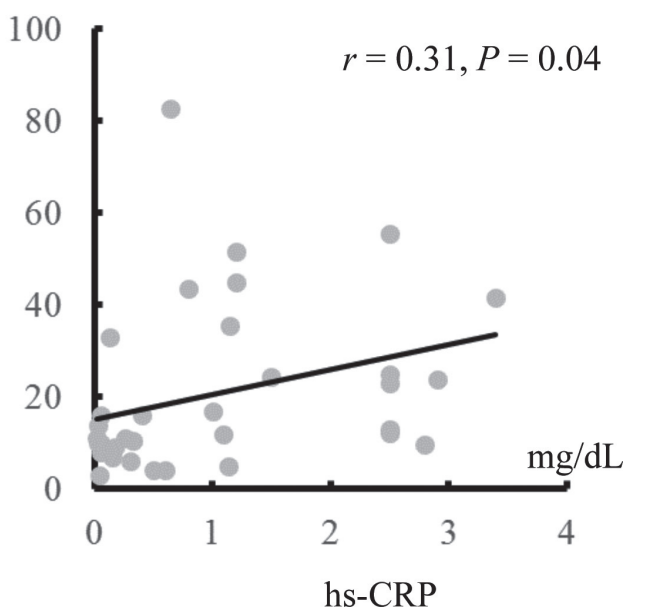

Figure 4. Correlation between percent of hs-CRP level between CD14-positive macrophages and CD163-positive macrophages. See text for details. 
cleared exclusively by CD163. ${ }^{14)}$ Therefore, the CD163-positive macrophage level is significantly greater in patients with ACS, and has a positive correlation with IPH area. ${ }^{5,15)}$ In addition, in coronary atherectomy specimens from patients with SAP or unstable angina pectoris (UAP), the CD163-positive macrophage score in UAP patients was significantly higher than that in SAP patients. ${ }^{15)}$ In the present study, the ACS group had a significantly higher \%CD163-positive macrophage level than the SAP group. This finding is in line with the above-mentioned findings. Therefore, in the present study, the accumulation of CD163-positive macrophages may reflect IPH.

CD14 on the macrophage surface was reported to be bound to lipopolysaccharide, thereby activating macrophages to produce cytokines such as TNF- $\alpha$, IL- 1 , and IL- 6 , which, in turn, serve as endogenous mediators of inflammation. ${ }^{3)}$ In a previous pathological report, CD14-positive macrophages were more prevalent in atherosclerotic lesions compared with normal intimal regions of coronary arteries. ${ }^{3)}$ Thus, CD 14positive macrophages within the plaque indicates plaque with inflammatory macrophage inflammation.

C-reactive protein, a laboratory marker of the systemic inflammatory status, is also synthesized at the site of the atherosclerotic plaque, and would reflect the destabilization process. ${ }^{16,11)}$ In vivo studies demonstrate that hs-CRP was associated with positive remodelling, fibrous cap thickness, and the presence of TCFA. ${ }^{17,18)}$ Furthermore, a higher prevalence of lesions with rupture ultrasonographic changes, with positive remodelling or lipid lakes was found in individuals with high CRP levels, as compared to patients with normal levels of this marker. ${ }^{19)}$ IPH is also an important contributor to lesion destabilization through mechanisms including extravasation of inflammatory factors such as macrophages and $\mathrm{Hb}$-derived iron. Therefore, in the present study, the hs-CRP level might have significant positive correlation with \%CD163-positive macrophages.

Difference in IVUS and VH-IVUS parameters between ACS and SAP: Positive remodeling is a compensatory enlargement to avoid a decrease in the coronary lumen. ${ }^{20)}$ Using IVUS, positive remodeling was observed frequently in patients with ACS compared to those with SAP. ${ }^{21)}$ Moreover, in both IVUS findings and pathological studies, plaque vulnerability associated with positive remodeling has also been demonstrated. ${ }^{22,23)} \mathrm{Ma}-$ trix metalloproteinases released into a plaque from macrophages may be involved in the development of positive remodeling. In an autopsy study, plaques with IPH and inflammation were more likely to undergo plaque expansion than plaques without these features. ${ }^{24)}$ Consistent with previous reports, the ACS group had a significantly higher level of \%CD163-positive macrophages and more positive remodeling than the SAP group in the present study.

Additionally, as compared with stable lesions with pathologic intimal thickening or early-core fibroatheroma, IPH was seen most frequently in vulnerable TCFA in an autopsy study. ${ }^{1)}$ Pathological data also shows that high-risk plaques such as vulnerable TCFA have more NC volume than stable plaques. ${ }^{25)}$ Furthermore, a previous report by IVUS also reported that ACS patients showed a significantly higher ratio of NC plaque compared with SAP patients, and VH-IVUS-derived TCFA was more frequently observed in ACS patients compared with SAP patients. ${ }^{26)}$ In the present study, the ACS group had signif- icantly more \% NC and $\mathrm{NC}$ volume and less \%Fibrous than the SAP group. Additionally, \%CD163-positive macrophages were positively correlated with $\% \mathrm{NC}$ and negatively correlated with \%Fibrous in culprit plaque. These findings may be consistent with those of the above-mentioned studies.

However, VH-IVUS data concerning the relationship of $\mathrm{NC}$ to clinical presentation are still controversial. ${ }^{25,27)}$ In $\mathrm{VH}-$ IVUS studies, the presence of thrombi in ACS lesions may influence plaque composition because thrombi are not recognized as a different component but assigned to one of the four available components (usually fibrous or fibro-fatty by $\mathrm{VH}$ IVUS). ${ }^{28)}$ Thus, it is possible that ACS may disturb accurate classification of the plaque components by VH-IVUS. To minimize this error, thrombi were intensively aspirated from culprit lesions before VH-IVUS examination in the present study. Limitations: The present study has several limitations. First, sampling bias may have affected the results. The use of thrombectomy itself can disturb plaque and the amount of collected debris may depend on the PCI operator since some may use a technique more likely to embolize material downstream. The debris represented a limited portion of the entire plaque. Additionally, CD14- and CD163-positive monocytes could be found in the blood. It was therefore difficult to distinguish blood-derived monocytes from plaque-derived macrophages in debris retrieved in the present study. However, histological examination revealed that about 75 percent of our debris contained cholesterol crystals, indicating this debris and macrophages that originated mainly from coronary plaque. ${ }^{29)}$ The incidence of cholesterol crystals within retrieved materials is consistent with a previous report. ${ }^{30)}$ Second, the present study had a relatively small sample size and excluded patients whose debris was not collected by Filtrap ${ }^{\circledR}$. Third, in VH-IVUS studies, the presence of thrombi in ACS lesions may influence the plaque composition. To minimize this error, thrombi were intensively aspirated from culprit lesions before VH-IVUS examination in the present study. ${ }^{31)}$ Finally, since this is a small observational study, further studies are needed to investigate the validity and the mechanism.

Conclusions: Although limited for these reasons, these findings suggest that the presence of CD14-positive macrophages and CD163-positive macrophages may reflect plaque inflammation, NC expansion, and plaque vulnerability in patients with coronary heart disease.

\section{Disclosure}

Competing interests: There are neither conflicts of interest nor funding to declare related to this work.

\section{REFERENCES}

1. Kolodgie FD, Gold HK, Burke AP, et al. Intraplaque hemorrhage and progression of coronary atheroma. N Eng J Med 2003; 349: 2316-25.

2. Libby P. Inflammation in atherosclerosis. Nature 2002; 420: 86874. (Review)

3. Waldo SW, Li Y, Buono C, et al. Heterogeneity of human macrophages in culture and in atherosclerotic plaques. Am J Pathol 2008; 172: 1112-26.

4. Boyle JJ, Harrington HA, Piper E, et al. Coronary intraplaque he- 
morrhage evokes a novel atheroprotective macrophage phenotype. Am J Pathol 2009; 174: 1097-108.

5. Philippidis P, Mason JC, Evans BJ, et al. Hemoglobin scavenger receptor CD163 mediates Interleukin-10 release and heme oxygenase-1 synthesis: anti-inflammatory monocyte-macrophage responses in vitro, in resolving skin blisters in vivo, and after cardiopulmonary bypass surgery. Circ Res 2004; 94: 119-26.

6. Virmani R, Kolodgie FD, Burke AP, et al. Atherosclerotic plaque progression and vulnerability to rupture: angiogenesis as a source of intraplaque hemorrhage. Arterioscler Thromb Vasc Biol 2005; 25: 2054-61. (Review)

7. Wu X, Maehara A, Mintz GS, et al. Virtual histology intravascular ultrasound analysis of non-culprit attenuated plaques detected by grayscale intravascular ultrasound in patients with acute coronary syndromes. Am J Cardiol 2010; 105: 48-53.

8. Sato T, Kameyama T, Noto T, et al. The impact of anti-inflammatory cytokines provoked by $\mathrm{CD} 163$ positive macrophages on ventricular functional recovery after myocardial infarction. J Thromb Thrombolysis 2014; 37: 139-47.

9. Mintz GS, Nissen SE, Anderson WD, et al. American College of Cardiology Clinical Expert Consensus Document on Standards for Acquisition, Measurement and Reporting of Intravascular Ultrasound Studies (IVUS). A report of the American College of Cardiology Task Force on Clinical Expert Consensus Documents. J Am Coll Cardiol 2001; 37: 1478-92. (Review)

10. Pasterkamp G, Borst C, Gussenhoven EJ, et al. Remodeling of De Novo atherosclerotic lesions in femoral arteries: impact on mechanism of balloon angioplasty. J Am Coll Cardiol 1995; 26: 422-8.

11. Nasu K, Tsuchikane E, Katoh O, et al. Accuracy of in vivo coronary plaque morphology assessment: a validation study of in vivo virtual histology compared with in vitro histopathology. J Am Coll Cardiol 2006; 47: 2405-12.

12. Michel JB, Virmani R, Arbustini E, Pasterkamp G. Intraplaque haemorrhages as the trigger of plaque vulnerability. Eur Heart J 2011; 32: 1977-985.

13. Boyle JJ, Johns M, Kampfer T, et al. Activating transcription factor 1 directs Mhem atheroprotective macrophages through coordinated iron handling and foam cell protection. Circ Res 2012; 110: 20-33.

14. Levy AP, Levy JE, Kalet-Litman S, et al. Haptoglobin genotype is a determinant of iron, lipid peroxidation, and macrophage accumulation in the atherosclerotic plaque. Arterioscler Thromb Vasc Biol 2007; 27: 134-40.

15. Yunoki K, Naruko T, Komatsu R, et al. Enhanced expression of haemoglobin scavenger receptor in accumulated macrophages of culprit lesions in acute coronary syndromes. Eur Heart J 2009; 30: 1844-52.

16. Nair A, Kuban BD, Tuzcu EM, Schoenhagen P, Nissen SE, Vince DG. Coronary plaque classification with intravascular ultrasound radiofrequency data analysis. Circulation 2002; 106: 2200-6.

17. Kashiwagi M, Tanaka A, Kitabata $\mathrm{H}$, et al. Feasibility of noninvasive assessment of thin-cap fibroatheroma by multidetector com- puted tomography. JACC Cardiovasc Imaging 2009; 2: 1412-9.

18. Kashiwagi M, Tanaka A, Kitabata H, et al. Relationship between coronary arterial remodeling, fibrous cap thickness and high-sensitivity C-reactive protein levels in patients with acute coronary syndrome. Circ J 2009; 73: 1291-5.

19. Sano T, Tanaka A, Namba M, et al. C-reactive protein and lesion morphologyin patients with acute myocardial infarction. Circulation 2003; 108: 282-5.

20. Glagov S, Weisenberg E, Zarins CK, Stankunavicius R, Kolettis GJ. Compensatory enlargement of human atherosclerotic coronary arteries. N Engl J Med 1987; 316: 1371-5.

21. Benedek T, Jako B, Benedek I. Plaque quantification by coronary $\mathrm{CT}$ and intravascular ultrasound identifies a low CT density core as a marker of plaque instability in acute coronary syndromes. Int Heart J 2014; 55: 22-8.

22. Pasterkamp G, Schoneveld AH, van der Wal AC, et al. Relation of arterial geometry to luminal narrowing and histologic markers for plaque vulnerability: the remodeling paradox. J Am Coll Cardiol 1998; 32: 655-62.

23. Varnava AM, Mills PG, Davies MJ. Relationship between coronary artery remodeling and plaque vulnerability. Circulation 2002; 105: 939-43.

24. Burke AP, Kolodgie FD, Farb A, Webber D, Virmani R. Morphological predictors of arterial remodeling in coronary atherosclerosis. Circulation 2002; 105: 297-303.

25. Surmely JF, Nasu K, Fujita H, et al. Coronary plaque composition of culprit/target lesions according to the clinical presentation: a virtual histology intravascular ultrasound analysis. Eur Heart J 2006; 27: 2939-44.

26. Nakamura T, Kubo N, Funayama H, Sugawara Y, Ako J, Momomura S. Plaque characteristics of the coronary segment proximal to the culprit lesion in stable and unstable patients. Clin Cardiol 2009; 32: E9-12.

27. Rodriguez-Granillo GA, García-García HM, Mc Fadden EP, et al. In vivo intravascular ultrasound-derived thin-cap fibroatheroma detection using ultrasound radiofrequency data analysis. J Am Coll Cardiol 2005; 46: 2038-42.

28. Nasu K, Tsuchikane E, Katoh O, et al. Impact of intramural thrombus in coronary arteries on the accuracy of tissue characterization by in vivo intravascular ultrasound radiofrequency data analysis. Am J Cardiol 2008; 101: 1079-83.

29. Angelini A, Della Barbera M, Thiene G. Interventinal procedures for atherothrombosis: pathology of retrieved material. Heart 2007; 93: 1301-8. (Review)

30. Kotani J, Nanto S, Mintz GS, et al. Plaque gruel of atheromatous coronary lesion may contribute to the no-reflow phenomenon in patients with acute coronary syndrome. Circulation 2002; 106: 1672-7.

31. Ito H. Etiology and clinical implications of microvascular dysfunction in patients with acute myocardial infarction. Int Heart $\mathbf{J}$ 2014; 55: 185-9. (Review) 